06

\title{
Влияние крутизны фронта импульса напряжения на электрическую прочность полимеров
}

\author{
(C) В.А. Закревский, ${ }^{1}$ В.А. Пахотин, ${ }^{1}$ Н.T. Сударь ${ }^{2, \text { ॠ }}$ \\ ${ }^{1}$ Физико-технический институт им. А.Ф. Иофффе РАН, \\ 194021 Санкт-Петербург, Россия \\ ${ }^{2}$ Санкт-Петербургский политехнический университет Петра Великого, \\ 195251 Санкт-Петербург, Россия \\ ฯ e-mail: sudar53@mail.ru
}

(Поступило в Редакцию 2 марта 2018 г.)

Исследовано влияние крутизны переднего фронта высоковольтного импульса на электрическую прочность пленки полиэтилентерефталата толщиной $3 \mu \mathrm{m}$. Установлено, что ее пробивная напряженность логарифмически возрастает с 550 до $700 \mathrm{MV} / \mathrm{m}$ при увеличении скорости нарастания напряжения (крутизны фронта импульса) с 2 до $70 \mathrm{GV} / \mathrm{s}$. Показано, что полученные экспериментальные данные можно описать, используя представление об ионизационном механизме пробоя полимеров, не связанном с развитием в них ударной ионизации и учитывая уменьшение коэффициента электрических перенапряжений в полимерном диэлектрике при увеличении крутизны фронта импульса.

DOI: $10.21883 /$ JTF.2019.01.46972.98-18

\section{Введение}

Актуальность исследования закономерностей пробоя полимерных диэлектриков при кратковременном (импульсном) воздействии на них сильного электрического поля обусловлена как потребностями современной импульсной техники, так и отсутствием единой точки зрения на физический механизм развития пробоя полимеров в этих условиях.

В современной импульсной технике (импульсных конденсаторах, трансформаторах и других устройствах) в качестве изоляции широко используются полимерные пленки, обладающие высокой электрической прочностью. Поэтому изучению электропрочностных свойств пленок полимеров уделяется большое внимание. Особый интерес представляет изучение электрической прочности полимеров в микро- и субмикросекундном диапазонах времен, когда время возрастания напряжения (длительность переднего фронта импульса) составляет $10-100$ ns. При этом скорость возрастания напряжения $d V / d t$ достигает значений в десятки GV/s. Поэтому необходимость изучения электропрочностных свойств полимеров в этих условиях не вызывает сомнений.

Известен ряд работ, в которых исследовалось влияние крутизны фронта импульса на величину пробивной напряженности поля $F_{b r}$ образцов различных полимерных диэлектриков [1-7].

В работах $[1,2]$ при изучении импульсного пробоя полиметилметакрилата было установлено, что при возрастании крутизны фронта импульса с 0.5 до $200 \mathrm{GV} /(\mathrm{m} \cdot \mathrm{s})$ значения $F_{b r}$ уменьшаются с 13 до $6 \mathrm{MV} / \mathrm{cm}$, но при крутизне фронта импульса менее $0.5 \mathrm{GV} /(\mathrm{m} \cdot \mathrm{s})$ зарегистрировано возрастание $F_{b r}$ при увеличении $d V / d t$, т. е. на зависимости $F_{b r}(d V / d t)$ наблюдался максимум. Напротив, наличие минимума пробивного напряжения на $F_{b r}(d V / d t)$ зафиксировано в работе [3] для таких полимеров как политетрафторэтилен, полиэтилентерефталат (ПЭТФ) и полиимид. Однако в работах $[4,6,7]$ для образцов полипропилена [4], ПЭТФ и полиэтилена [6], акриловой ленты VHB [7] на зависимости $F_{b r}(d V / d t)$ зафиксировано только увеличение $F_{b r}$ при увеличении крутизны фронта импульса напряжения.

Цитируемые выше публикации, несмотря на противоречивость представленных в них данных, свидетельствуют о существенном влиянии крутизны фронта импульса на импульсную электрическую прочность полимеров.

Целью настоящей работы являлось изучение влияния крутизны фронта импульса, т.е. скорости приложения электрического поля к пленочным полимерным диэлектрикам, на их электрическую прочность и рассмотрение результатов соответствующих экспериментов с позиций современных представлений о процессах электрического пробоя полимерных диэлектриков.

\section{Методика эксперимента}

Объектом исследования являлась двуосноориентированная пленка ПЭТФ промышленного производства толщиной $d=3 \mu \mathrm{m}$. Она закреплялась в специальной оправке между двумя стальными электродами и помещалась в конденсаторное масло. Один из электродов (плоский) находился под потенциалом земли. На второй (сферический электрод диаметром $6 \mathrm{~mm}$ ) подавался высоковольтный импульс отрицательной полярности длительностью $\sim 10 \mu \mathrm{s}$. Напряжение на его переднем фронте изменялось со временем по закону

$$
V(t)=V_{m}\left[1-\exp \left(-\frac{t}{\tau}\right)\right],
$$

где $V_{m}$ - амплитуда импульса $\left(V_{m}=2500 \mathrm{~V}\right), t$ - время, $\tau$ - постоянная времени. Генератор высоковольтных 
импульсов обеспечивал возможность варьирования значения $\tau$ в диапазоне $10^{1}-10^{3} \mathrm{~ns}$. В качестве параметра, характеризующего крутизну фронта импульса, в дальнейшем использовалось отношение $V_{m} / \tau$.

Испытание пленки на пробой заключалось в однократном воздействии на нее высоковольтного импульса с фиксированным для каждой серии испытаний параметром $V_{m} / \tau$. В каждой серии испытывалось 30 образцов. Все испытания проводились при температуре $293 \mathrm{~K}$.

Для измерения напряжения на образце и определения момента электрического пробоя использовался широкополосный запоминающий осциллограф ADS2332 (интервал дискретизации $1 \mathrm{~ns}$ ) с высоковольтным (до $2.5 \mathrm{kV}$ ) широкополосным делителем TTHV250 с граничной частотой $300 \mathrm{MHz}$. Момент пробоя пленки на осциллограммах фиксировался по резкому падению напряжения на образце.

\section{Экспериментальные данные и их обсуждение}

В ходе электрических испытаний было установлено, что при однократном воздействии на образец пленки ПЭТФ высоковольтного импульса электрический пробой мог наблюдаться как на его фронте, так и плато. Для некоторых образцов при выбранной величине $V_{m}$ пробой не регистрировался вовсе. На рис. 1 представлены распределения долей образцов (в процентах) по отношению к общему числу образцов в серии, соответствующие пробою на фронте и на плато импульса, а также отсутствию пробоя при различных значениях крутизны фронта импульса. Видно, что пробой происходит в основном на фронте импульсов. При этом доля образцов возрастает по мере уменьшения крутизны фронта, т.е. по мере увеличения времени воздействия возрастающего напряжения, достигая значения $\sim 96 \%$ при $2 \mathrm{GV} / \mathrm{s}$. Таким образом, так же как и в работе [8], можно сделать вывод о том, что часть образцов „не успевает“

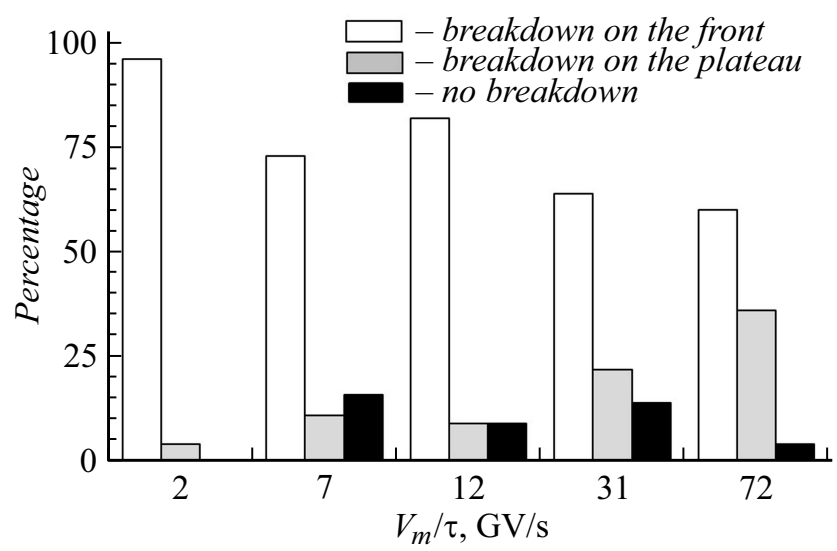

Рис. 1. Доли образцов в процентах по отношению к общему числу образцов в серии, соответствующие пробою на фронте и плато импульса, а также отсутствию пробоя образца при различных величинах $V_{m} / \tau$.

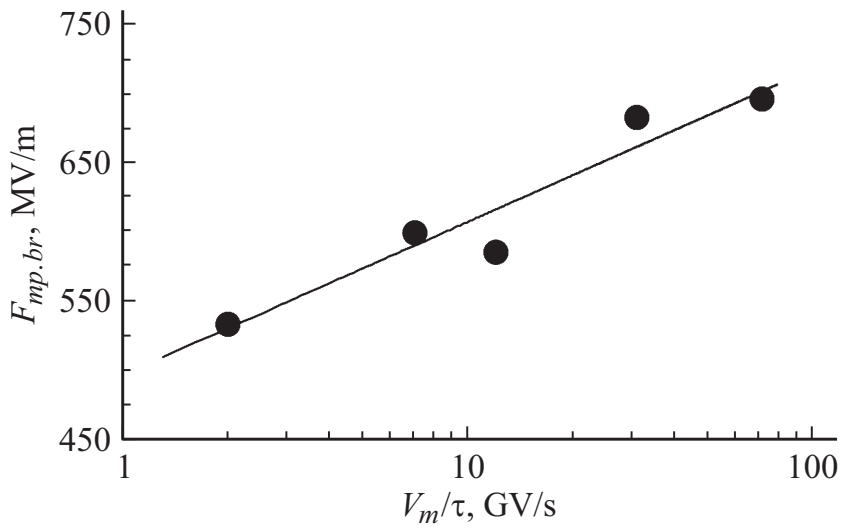

Рис. 2. Зависимость наиболее вероятной пробивной напряженности пленки ПЭТФ от величины $V_{m} / \tau$.

пробиться за время воздействия импульса с указанной длительностью, амплитудой и крутизной фронта. Подобные результаты изучения воздействия импульсного напряжения на полимеры получены в работах $[8,9]$. Анализу результатов, полученных при пробое на фронте импульсов, далее будет уделено основное внимание.

Рассмотрим данные, характеризующие особенности пробоя на фронте импульсов, т.е. данные о зависимости электрической прочности полимеров от скорости подъема напряжения. При пробое пленки на фронте импульса значения величин пробивного напряжения $V_{b r}$ характеризуются значительным разбросом, что позволяет рассматривать значения $V_{b r}$ как случайные величины, реализуемые с определенной вероятностью. При статистическом анализе экспериментальных результатов исследования пробоя полимерной пленки использовалось двухпараметрическое распределение Вейбулла, имеющее вид

$$
f\left(V_{b r}\right)=1-\exp \left[-\left(\frac{V_{b r}}{V_{0}}\right)^{\beta}\right],
$$

где $f\left(V_{b r}\right)$ - вероятность того, что пробой произойдет при напряжении $V_{b r}, \beta-$ параметр формы, $V_{0}-$ параметр масштаба распределения.

Результаты аппроксимации эмпирической функции распределения распределением Вейбулла представлены в таблице. Отметим, что достоверность аппроксимации $r^{2}$ результатов испытаний распределением Вейбулла превышает 0.92 для всех значений крутизны фронта импульса, что свидетельствует об обоснованности его применения. Определив на основании экспериментальных данных параметры $\beta$ и $V_{0}$, можно рассчитать наиболее вероятное значение пробивного напряжения $V_{m p . b r}$ и дисперсию распределения [10].

На рис. 2 представлена зависимость наиболее вероятной пробивной напряженности $F_{m p . b r}\left(F_{m p . b r}=V_{m p . b r} / d\right)$ от $V_{m} / \tau$ для пленки ПЭТФ. Оценки величины ошибок для значений $F_{m p . b r}$ (на основании данных таблицы) приводят к значениям коэффициента вариации $\sim 30 \%$. Представленные результаты испытаний на пробой позволяют сделать вывод о том, что в рассматриваемом 
Значения параметров распределения Вейбулла, используемого для описания $f\left(V_{b r}\right)$, и коэффициент корреляции $r^{2}$ при различных значениях $V_{m} / \tau$

\begin{tabular}{c|c|c|c|c|c}
\hline$V_{m} / \tau, \mathrm{GV} / \mathrm{s}$ & 2 & 7 & 12 & 31 & 72 \\
\hline$\beta$ & 2.89 & 2.40 & 3.53 & 2.53 & 2.87 \\
\hline$V_{0}, \mathrm{~V}$ & 1797 & 2031 & 1953 & 2310 & 2343 \\
\hline$r^{2}$ & 0.98 & 0.98 & 0.95 & 0.92 & 0.97
\end{tabular}

диапазоне изменения $V_{m} / \tau$ увеличение крутизны фронта импульса приводит к возрастанию $F_{m p . b r}$ полимерной пленки с 550 до $700 \mathrm{MV} / \mathrm{m}$.

Рассматриваемая зависимость в полулогарифмических координатах $F_{m p . b r}-\lg \left(V_{m} / \tau\right)$ может быть аппроксимирована прямой

$$
F_{m p . b r}=a \lg \left(\frac{V_{m}}{\tau}\right)+b
$$

Значения параметров прямой (3), определенные с помощью метода наименьших квадратов, равны: $a=109.9 \mathrm{MV} / \mathrm{m}$ и $b=497.4 \mathrm{MV} / \mathrm{m}$. Здесь и далее при расчетах принималось $\left[V_{m} / \tau\right]=\mathrm{GV} / \mathrm{s}$.

Перейдем к обсуждению результатов экспериментов. В полимерных диэлектриках механизм ударной ионизации не может реализоваться [11-13]. Будем рассматривать в качестве причины электрического пробоя полимерных диэлектриков при воздействии на них импульсного напряжения полевую ионизацию макромолекул. Соответствующий физический механизм разрушения полимеров в электрическом поле, не связанный с ударной ионизацией, позволяет с единых позиций рассмотреть старение полимеров в электрическом поле со временем и объяснить быстрое возрастание тока при электрическом пробое $[13,14]$. Накопление в полимерах электронов и положительных молекулярных ионов (дырок) вследствие полевой ионизации макромолекул приводит к возникновению дебаевского экранирования зарядов и, как следствие этого, снижению потенциала ионизации молекул, что, в свою очередь, ускоряет образование несвязанных зарядов в полимере. Таким образом, реализуется самоускоряющийся процесс, приводящий к возрастанию концентрации свободных носителей зарядов. На определенном этапе происходит взрывообразное изменение свойств полимера - потеря им диэлектрических свойств, т. е. его пробой.

Ионизационный механизм электрического пробоя полимерных диэлектриков предполагает протекание двух взаимосвязанных процессов, обусловливающих возникновение в полимере твердотельной плазмы и увеличение ее концентрации до критического значения. В качестве таковых рассматривается полевая ионизация макромолекул и термофлуктуационный распад образующихся молекулярных ионов. Вследствие кратковременности развития импульсного пробоя можно ограничиться анализом лишь наиболее быстропротекающего процесса, а именно рассмотрением полевой ионизации макромолекул при туннельном переходе электрона с уровня высшей занятой молекулярной орбитали макромолекулы (HOMO) на низшую свободную орбиталь (LUMO) другой молекулы [8]. Такой подход был использован нами и в настоящей работе.

Отметим, что образовавшиеся в результате этих переходов возбужденные состояния являются межмолекулярными $C T$-состояниями [15]. Они являются предшественниками свободных носителей зарядов, возникающих при диссоциации таких состояний с переносом заряда:

$$
M_{1}+M_{2} \stackrel{F}{\rightarrow}\left(M_{1}^{+} M_{2}^{-}\right) \stackrel{F}{\rightarrow} M_{1}^{+}+M_{2}^{-} .
$$

Здесь $M_{1}$ и $M_{2}$ - макромолекулы, $M_{1}^{+}$и $M_{2}^{-}-$молекулярные ионы, $F$ - локальная напряженность электрического поля, действующего на молекулы. Диссоциация в электрическом поле рассматриваемых пар происходит с большой вероятностью, если $F>e /\left(4 \pi \varepsilon \varepsilon_{0} r^{2}\right.$, где $e-$ заряд электрона, $\varepsilon$ - диэлектрическая проницаемость полимера, $\varepsilon_{0}=8.85 \cdot 10^{-12} \mathrm{~F} / \mathrm{m}, r-$ расстояния между зарядами в паре. В сильных электрических полях это условие выполняется.

В этом случае скорость накопления дырок определяется уравнением

$$
\frac{d p}{d t}=k_{H L}(p)\left[M_{0}-p\right]
$$

где $p-$ концентрация дырок, $M_{0}-$ начальная концентрация способных ионизоваться фрагментов макромолекул, $k_{H L}(p)$ - константа скорости туннельной ионизации, обусловленной переходами HOMO-LUMO. Полагая, что электроны туннелируют в направлении электрического поля, а барьер является треугольным, определим $k_{H L}(p)$ как

$$
k_{H L}(p)=v_{0} \exp \left(-\frac{4 \sqrt{2 m}}{3 e \hbar} \frac{\Delta^{3 / 2}}{F}\right),
$$

где $v_{0}$ - частотный фактор, $v_{0} \approx 10^{16} \mathrm{~s}^{-1}, m-$ масса электрона, $\hbar$ - постоянная Планка, $\Delta-$ высота потенциального барьера.

С учетом снижения высоты потенциального барьера вследствие эффекта дебаевского экранирования для расчета $\Delta$ используем соотношение

$$
\Delta=E_{g}-\Delta I(p)
$$

Здесь $E_{g}$ - ширина энергетической щели, определяемая как $E_{g}=\left|E_{\mathrm{HOMO}}-E_{\mathrm{LUMO}}\right|\left(E_{\mathrm{HOMO}}\right.$ и $E_{\mathrm{LUMO}}-$ энергии уровней HOMO и LUMO), $\Delta I(p)-$ снижение энергии ионизации макромолекул вследствие эффекта дебаевского экранирования. Величина $\Delta I(p)$ рассчитывается по 


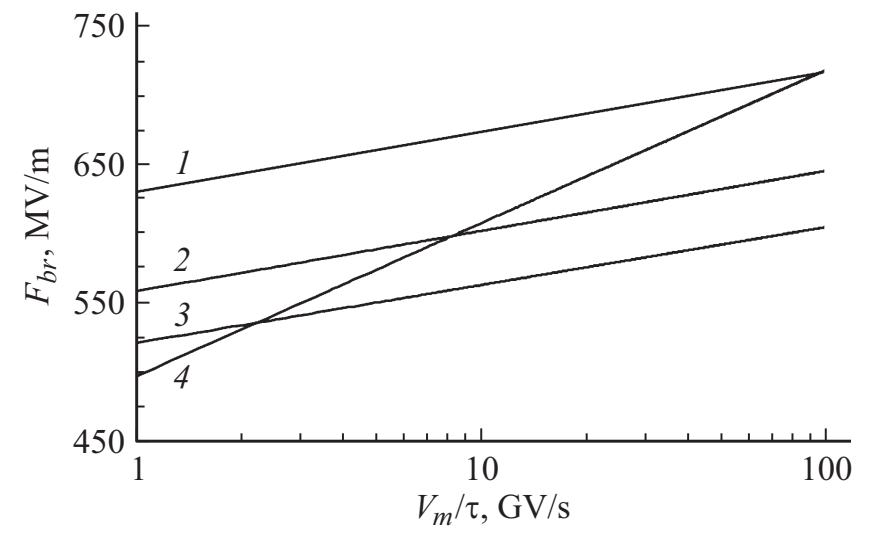

Рис. 3. Расчетные зависимости $F_{b r}\left(V_{m} / \tau\right)$, выполненные при значениях коэффициента электрических перенапряжений 5.7 (1), 6.6 (2) и 7.0 (3). Прямая 4 аппроксимирующая прямая экспериментальной зависимости $F_{m p . b r}\left(V_{m} / \tau\right)$ (тождественна прямой, изображенной на рис. 2).

формуле $[14,16]$

$$
\Delta I(p)=\frac{e^{2}}{4 \pi \varepsilon_{0} r_{D}(p)},
$$

где $r_{D}(p)$ - дебаевский радиус экранирования, равный

$$
r_{D}(p)=\sqrt{\frac{\varepsilon \varepsilon_{0} k_{B} T}{e^{2} p}},
$$

где $k_{B}-$ постоянная Больцмана, $T-$ температура.

Уравнение (4) решалось численно методом АдамсаBDF с начальным условием $p=0$ при $t=0$ [17]. Расчеты проводились при $V_{m}=2500 \mathrm{~V}, d=3 \mu \mathrm{m}, \varepsilon=3.1$, $M_{0}=10^{21} \mathrm{~cm}^{-3}$ и $E_{g}=6.5 \mathrm{eV}$ для различных значений $\tau$ при $T=293 \mathrm{~K}$. Принималось, что локальная напряженность поля $F$ изменяется со временем по закону $F(t)=k V(t) / d$, где $k-$ коэффициент электрических перенапряжений, а $V(t)$ - определяется соотношением (1). Момент времени пробоя, а следовательно и величина $F_{b r}$, определялись по моменту достижения критического значения концентрации дырок $p_{c r}=2 \cdot 10^{17} \mathrm{~cm}^{-3}[8]$. При такой концентрации дырок вследствие самоускоряющегося процесса полевой ионизации молекул происходит взрывообразное возрастание концентрации носителей зарядов. В результате наблюдается резкий рост тока, вызывающего пробой диэлектрика, что согласуется с результатами экспериментов по пробою полимеров [18].

Результаты расчетов зависимостей $F_{b r}\left(V_{m} / \tau\right)$, выполненных при $T=293 \mathrm{~K}$ и $k$, равных 5.7 (1), 6.5 (2) и 7.0 (3), представлены на рис. 3. Оказалось, что в полулогарифмических координатах они линейны, т.е.

$$
F_{b r}=a^{\prime} \lg \left(\frac{V_{m}}{\tau}\right)+b^{\prime}
$$

где $a^{\prime}$ и $b^{\prime}$ - параметры расчетных прямых.
Прямая 4 на этом рисунке соответствует зависимости $F_{m p . b r}\left[\lg \left(V_{m} / \tau\right)\right]$, построенной на основании экспериментально определенных значений параметров $a$ и $b$. Она тождественна прямой, изображенной на рис. 2.

Обращает на себя внимание различие в величинах углов наклона экспериментальной и расчетных прямых. Среднее значение величины $a^{\prime}$ расчетных прямых при изменении $k$ в интервале от 5.7 до 7 равно $(42.1 \pm 0.4) \mathrm{MV} / \mathrm{m}$, т.е. для расчетных прямых наклон оказался в $\sim 2.5$ раза меньше, чем для экспериментальной. Поэтому при $k=5.7$ наблюдается хорошее согласие экспериментальных и расчетных данных для импульсов с высокой крутизной фронта, для которых скорость возрастания напряжения составляет $\sim 10^{2} \mathrm{GV} / \mathrm{s}$, а при $k=7.0$ экспериментальные и расчетные данные согласуются при значениях $V_{m} / \tau$, равных $2-3 \mathrm{GV} / \mathrm{s}$, т.е. для импульсов с относительно невысокой крутизной фронта.

Расчеты показали, что по мере уменьшения величины $k$ прямые, определяемые соотношением (9), смещаются вверх по оси ординат так, что $a^{\prime} \approx$ const, а параметр прямых $b^{\prime}$ зависит от коэффициента электрических перенапряжений, причем в рассматриваемом интервале изменения $V_{m} / \tau$ реализуется соотношение

$$
b^{\prime}=b_{0}-\alpha k,
$$

где $b_{0}=1134 \mathrm{MV} / \mathrm{m}$ и $\alpha=87.6 \mathrm{MV} / \mathrm{m}$.

Ранее автором работы [1] было высказано предположение о причине влияния крутизны фронта импульса на пробивную напряженность электрического поля в полимерном диэлектрике. По его мнению, причиной этого влияния является накапливающийся в полимере объемный заряд (О3), влияющий на распределение электрического поля в полимере. Вопрос о том, как конкретно О3 влияет на $F_{b r}$ при различной скорости нагружения полимеров, в работе [1] не рассматривался.

Представленные на рис. 3 результаты показывают, что коэффициент усиления поля $k$ изменяется при изменении скорости подъема напряжения на образце, что несомненно связано с особенностями формирования О3, а именно с зависимостью этого процесса от напряжения и времени. Однако в связи с существованием зависимости $k\left(V_{m} / \tau\right)$ неясно, почему экспериментальная зависимость $F_{m p . b r}\left[\lg \left(V_{m}\right)\right]$ оказывается близка к линейной. Для ответа на этот вопрос проанализируем возможное влияние О3 на значение $k$ при импульсном воздействии на полимер более подробно, оценив величины $k$ при различных величинах крутизны фронта импульса.

В сильных электрических полях носители заряда (как электроны, так и дырки) способны инжектироваться из электродов в полимерный диэлектрик. Захватываясь на ловушки, они формируют в диэлектрике ОЗ. Отличительной особенностью полимерных диэлектриков является высокая концентрация в них нейтральных ловушек электронов $N_{t}$, достигающая значений $10^{18}-10^{19} \mathrm{~cm}^{-3}$ [19-21]. Примем при дальнейших расчетах $N_{t}=10^{18} \mathrm{~cm}^{-3}$. Формирование О3 происходит в 
результате конкуренции процессов захвата и освобождения электронов из ловушек. Среднее время удержания электрона на ловушке $t_{\text {trap }}$ при данной температуре определяется ее глубиной $E_{t}$ и равно

$$
t_{\text {trap }}=\tau_{0} \exp \left(\frac{E_{t}}{k_{B} T}\right),
$$

где $\tau_{0} \approx 10^{-13} \mathrm{~s}$.

Оценим концентрацию ловушек, способных удержать носители заряда в течение времени $t \geq t_{\text {trap. }}$ Из соотношения (11) следует, что данному условию удовлетворяют ловушки, глубина которых $E_{t}^{\prime} \geq k_{B} T \ln \left(t_{\text {trap }} / \tau_{0}\right)$.

Будем считать, согласно [22], что энергетическая плотность ловушечных состояний характеризуется экспоненциальным распределением

$$
g(E)=\frac{N_{t}}{\left\langle E_{t}\right\rangle} \exp \left(-\frac{E_{t}}{\left\langle E_{t}\right\rangle}\right)
$$

где $\left\langle E_{t}\right\rangle-$ средняя глубина ловушек, $\left\langle E_{t}\right\rangle=0.5 \mathrm{eV}$. Концентрация ловушечных состояний $n_{t}$, которые в данных условиях можно рассматривать как „глубокие“, занятые носителями заряда ловушки, определяется посредством интегрирования соотношения (12) в пределах от $E_{t}^{\prime}$ до $\infty$. Для $T=293 \mathrm{~K}$ при изменении $t_{\text {trap }}$ от 10 до $10^{3} \mathrm{~ns}$ значение $n_{t}$ изменяется от $0.55 N_{t}$ до $0.44 N_{t}$.

Столь слабая зависимость $n_{t}$ от $t_{\text {trap }}$ позволяет моделировать процесс накопления О3 как прорастание в глубь полимера слоя О3 с постоянной в пространстве и времени концентрацией, равной примерно $0.5 N_{t}$.

Оценим достигаемые значения $k$ и время накопления O3 вблизи от инжектирующего электрода в плоскопараллельной электродной системе. Примем, что плотность распределения заряда $n_{t}(x)$ в межэлектродном пространстве определяется как

$$
n_{t}(x)= \begin{cases}0.5 N_{t}, & \text { если } 0 \leq x \leq x_{f} \\ 0, & \text { если } x_{f} \leq x \leq d\end{cases}
$$

Здесь $x_{f}$ - граница прорастания заряда, а $x=0$ соответствует поверхности электрода.

Решая уравнение Пуассона для поля $F(x)$ и заряда $n_{t}(x)$ и полагая, что $\int_{0}^{d} F(x) d x=V$, получим, что максимальная напряженность электрического поля $F_{\max }$ достигается при $x \geq x_{f}$ и при $x_{f} \ll d$ равна

$$
F_{\max } \approx F_{a v}+\frac{e N_{t} x_{f}}{2 \varepsilon \varepsilon_{0}}
$$

где $F_{a v}-$ средняя напряженность поля в диэлектрике, равная $F_{a v}=V / d$. Из соотношения (14) следует, что $k=F_{\max } / F_{a v}$ возрастает по мере увеличения глубины прорастания О3 и его концентрации. Оценим глубину прорастания заряда в зависимости от крутизны фронта импульса. Учтем, что в экспериментах в момент пробоя $F_{a v}=F_{b r}$. Примем значения $k$ равными 5.7 и 7 , т.е. будем полагать их равными значениям $k$, используемым ранее при построении прямых 1,3 на рис. 3. Тогда из соотношения (14) получим, что граница прорастания заряда слабо зависит от крутизны фронта импульса и составляет $\sim 0.2 \mu \mathrm{m}$, хотя время воздействия электрического поля (время до пробоя) отличается при этом в 100 раз.

Оценим время $\Delta t$, за которое заряд может прорасти на глубину $0.2 \mu \mathrm{m}$. Будем считать, что $x_{f} \approx \mu F_{\max } \Delta t$, где $\mu-$ подвижность носителей заряда. Примем, согласно [18], $\mu \approx 10^{-8} \mathrm{~m}^{2} /(\mathrm{V} \cdot \mathrm{s})$, а $F_{\max } \approx k F_{b r}$. Тогда при $k=5.7$ (это значение $k$ соответствует крутизне фронта импульса $\sim 100 \mathrm{GV} /(\mathrm{m} \cdot \mathrm{s}))$, получим $\Delta t \approx 6 \mathrm{~ns}$. Поэтому даже при самой высокой скорости подъема напряжения, реализуемой в наших экспериментах и, как следствие этого, минимальном времени воздействия электрического поля, к моменту пробоя в полимерном диэлектрике успевает накопиться О3 с высокой концентрацией и сформироваться область электрических перенапряжений.

Изложенное выше дает основание предполагать, что процесс накопления О3 в полимерном диэлектрике при воздействии на него высоковольтных импульсов развивается по следующей схеме. Первоначально в течение нескольких наносекунд в приэлектродной области полимерного диэлектрика накапливается О3, характеризующийся высокой концентрацией. Глубина его прорастания невелика и составляет десятые доли микрона. В области $x \geq x_{f}$ поле усиливается, достигая значений, при которых становится возможной полевая ионизация молекул. Однако в дальнейшем вследствие ограничения тока инжекции, накопление О3 резко замедляется. При увеличении $\tau$ (уменьшении крутизны фронта импульса) время до пробоя возрастает, что и приводит к некоторому увеличению глубины прорастания заряда и, как следствие этого, к увеличению напряженности электрического поля в области $x \geq x_{f}$.

Отметим, что взаимосвязь между $k$ и $V_{m} / \tau$, которую необходимо учитывать для согласования расчетных и экспериментальных данных, с одной стороны, должна соответствовать линейному характеру зависимости $F_{b r}\left[\lg \left(V_{m} / \tau\right)\right]$, а с другой - учитывать рассмотренную выше качественную схему накопления ОЗ. Этим требованиям удовлетворяет следующее соотношение:

$$
k \approx k_{0}-\gamma \lg \left(\frac{V_{m}}{\tau}\right),
$$

где $k_{0}$ и $\gamma$ - положительные константы. Параметр $k_{0}$ соответствует значению коэффициента перенапряжений при времени инжекции менее $10 \mathrm{~ns}$, а параметр $\gamma$ определяет процесс изменения значения $k$ со временем. Действительно, преобразовав выражение (9) с учетом соотношений (10) и (15), получим

$$
F_{b r}=\left(a^{\prime}+\alpha \gamma\right) \lg \left(\frac{V_{m}}{\tau}\right)+\left(b_{0}-\alpha k_{0}\right) .
$$

Из соотношения (16) следует, что учет взаимосвязи между $k$ и $V_{m} / \tau$ в виде (15) при расчете пробивной 
напряженности полимерной пленки, не изменяет формы зависимости $F_{b r}\left[\lg \left(V_{m} / \tau\right)\right]$, а приводит лишь к увеличению угла наклона расчетной прямой.

Наилучшее соответствие между расчетными и экспериментальными данными достигается при $k_{0}=7.1$ и $\gamma=0.82$. В этом случае $\left(a^{\prime}+\alpha \gamma\right)=114.3 \mathrm{MV} / \mathrm{m}$ и $\left(b_{0}-\alpha k_{0}\right)=509.2 \mathrm{MV} / \mathrm{m}$, которые хорошо согласуется с параметрами $a$ и $b$, определенными из экспериментов.

\section{Заключение}

Таким образом, в настоящей работе получены экспериментальные данные о влиянии крутизны фронта высоковольтных импульсов на электропрочностные свойства пленки ПЭТФ. Установлено, что пробивная напряженность пленки ПЭТФ логарифмически возрастает с ростом скорости повышения напряжения. Показано, что полученные результаты можно описать, используя представления об ионизационном механизме пробоя полимеров, не связанном с развитием в них ударной ионизации. Наблюдаемое возрастание $F_{b r}$ исследуемой пленки при увеличении крутизны фронта импульса в рассматриваемом диапазоне изменения $V_{m} / \tau$ может быть связано с двумя факторами, а именно изменением скорости накопления ионизированных фрагментов макромолекул и уменьшением коэффициента электрических перенапряжений в полимерном диэлектрике при увеличении крутизны фронта импульса.

\section{Список литературы}

[1] Watson D.B. // J. Phys. D: Appl. Phys. 1972. Vol. 5. P. 410414. DOI: $10.1088 / 0022-3727 / 5 / 2 / 323$

[2] Watson D.B. // J. Phys. D: Appl. Phys. 1971. Vol. 4. P. 47-48. DOI: $10.1088 / 0022-3727 / 4 / 5 / 101$

[3] Важсов В.Ф., Молдобаев К.Д. // Электричество. 2009. № 12. C. $89-92$.

[4] Wilson M.P., Timoshkin I.V., Given M.J., Macgregor S.J., Sinclair M.A., Thomas K.J., Lehr J.M. // IEEE Trans. Elect. Insul. 2011. Vol. 18. N 4. P. 1003-1010. DOI: 10.1109/TDEI.2011.5976088

[5] Kitani I., Arii K. // IEEE T. Elect. Insul. 1980. Vol. 15. N 2. P. 134-139. DOI: 10.1109/TEI.1981.298353

[6] Кривко В.В., Лехт Ю.И. // Изв. ТПУ. 1970. Т. 157. С. 201205.

[7] Смирнов И.В., Springhetti R., Морозов В.А., Лукин А.А. // ЖТФ. 2018. Т. 88. Вып. 1. С. 151-153. DOI: $10.21883 /$ JTF.2018.01.45500.2177

[8] Закревский В.А., Пахотин В.А., Сударь Н.Т. // ЖТФ. 2017. T. 87. Вып. 2. C. 249-253. [Zakrevskii V.A., Pakhotin V.A., Sudar' N.T. // Tech. Phys. 2017. Vol. 62. N 2. P. 276-281.] DOI: 10.21883/JTF.2017.02.44133.1907

[9] Yamada H., Fujiwara T., Suzuoki Y. // J. Phys. D: Appl. Phys. 1993. Vol. 26. P. $1328-1330$. DOI: $10.1088 / 0022-3727 / 26 / 8 / 029$

[10] Капур К., Ламберсон Л. Надежность и проектирование систем. М.: Мир, 1980. 604 с.
[11] Dissado L.A., Fothergill J.C. Electrical Degradation and Breakdown in Polymers. London: Peter Peregrinus, 1992. $620 \mathrm{p}$.

[12] Закревский В.А., Сударь Н.T. // ФТТ. 2005. Т. 47. Вып. 5. C. 931-936. [Zakrevskii V.A., Sudar' N.T. // Phys. Solid State. 2005. Vol. 47. N 5. P. 961-967.]

[13] Закревский В.А., Сударь Н.T. // ФТТ. 2013. Т. 55. Вып. 7. C. 1298-1303. [Zakrevskii V.A., Sudar' N.T. // Phys. Solid State. 2013. Vol. 55. N 7. P. 1395-1400.

[14] Zakrevskii V.A., Pakhotin V.A., Sudar N.T. // J. Appl. Phys. 2014. Vol. 115. P. 234101. DOI: $10.1063 / 1.4883365$

[15] Dyakonov V., Frankevich E. // Chem. Phys. 1998. Vol. 227. N 1-2. P. 203-217. DOI: 10.1016/S0301-0104(97)00305-4

[16] Griem H.R. // Phys. Rev. 1962. Vol. 128. N 3. P. 997-1003. DOI: $10.1103 /$ PhysRev.128.997

[17] Кирьянов Д.Д. Mathcad 14. СПб.: БХВ-Петербург, 2006. $682 \mathrm{c}$.

[18] Hikita M., Kanno I., Sawa G., Ieda M. // Jpn. J. Appl. Phys. 1985. Vol. 24. Pt 1. N 8. P. 984-987. DOI: 10.1143/JJAP.24.984

[19] Тютнев А.П., Садовничий Д.Н., Саенко В.С., Пожидаев Е.Д. // Высокомолекулярные соед. Сер. А. 2000. Т. 42. № 1. C. 16-26. [Tyutnev A.P., Saenko V.S., Pozhidaev E.D., Sadovnichii D.N. // Polym. Sci. Ser. A. 2000. Vol. 42. N 1. P. 10-18.]

[20] Anta J.A., Marcelli G., Meunier M., Quirke N. // J. Appl. Phys. 2002. Vol. 92. N 2. P. $1002-1008$. DOI: $10.1063 / 1.1489714$

[21] Meunier M., Quirke N. // J. Chem. Phys. Vol. 113. N 1. P. 369-376. DOI: $10.1063 / 1.481802$

[22] Тютнев А.П., Саенко В.С., Пожидаев Е.Д., Костюков Н.С. Диэлектрические свойства полимеров в полях ионизирующих излучений. М.: Наука, 2005. 453 с. 